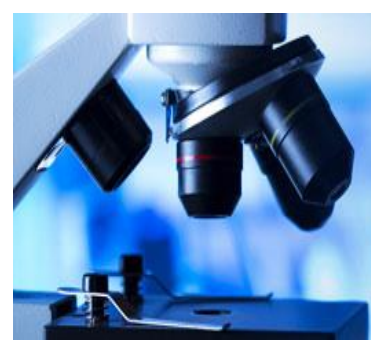

E-ISSN: 2707-4455

P-ISSN: 2707-4447

IJFM 2020; 2(2): 21-24

Received: 15-05-2020

Accepted: 19-07-2020

\section{Mansi Chauhan}

M.Sc. Forensic Science 4th

Semester Final Year, School of Basic Applied and Science,

Galgotias University, Greater

Noida, Uttar Pradesh, India
Corresponding Author: Mansi Chauhan

M.Sc. Forensic Science 4th

Semester Final Year, School of

Basic Applied and Science,

Galgotias University, Greater

Noida, Uttar Pradesh, India

\title{
Storage of saliva and blood specimen in different temperature
}

\section{Mansi Chauhan}

DOI: https://doi.org/10.33545/27074447.2020.v2.i2a.28

Abstract

In forensic sciences, Blood and saliva are two of the most examined biological samples used in forensic toxicology as they essentially contains all the information (in the form of DNA as well as certain other chemical compounds like salivary amylase etc.) that is needed to narrow down possible suspects and also to determine the identity of the true criminal. There is a time gap between collection of blood from the criminal site and its analysis in laboratory so it is very important to store and handle the biological specimen appropriately in order to maintain its stability. This paper presents a review on storage of biological sample mainly blood and saliva in different temperature.

Keywords: Blood, saliva, post mortem and ante mortem blood sample, preservation and storage

\section{Introduction}

Storage of biological sample is one of the most important steps in forensic toxicological investigation. The stability of a collected sample from the crime scene depends on its storage and according to that its impact on the forensic analysis. Various changes have been seen while collecting postmortem specimen. These changes may be autolytic and putrefactive in nature. Easiness of using and handling the specimen, its volume and putrefactive etc. are some of the parameter that should be considered while selecting specimen.

\section{Specimen container}

Choice of container depends on the specimen like its weight and volume. Many specimen get adhere to the glass and plastic surfaces hence in interfere the forensic case work. In forensic science glass tubes are generally used for ante mortem and post mortem blood sampling. Different additives are used to stabilize the blood specimen in these glass tubes like sodium fluoride.

\section{Blood}

In most of the criminal cases blood is the most important specimen for forensic analysis. There are various methods present in forensic toxicology to analyze blood. Blood is one of the critically used specimens in determining concentration of drug in both post mortem and ante mortem cases.

Blood is used as ante mortem biological sample because of its widely accepted matrix, used in determination of drug concentration in blood and its related pharmacological effects. Moreover blood is not adulterer readily hence give can be used to give extensive reference data. One of the disadvantage of using blood as ante mortem specimen is the shorter detection time.

Blood is used as post mortem biological sample because blood collected from peripheral and central nervous system helps to estimate the concentration of drug in blood sample. There are several downfall of using blood as postmortem biological sample as the collection of blood from peripheral part of body require expert individual. Moreover only limited volume of peripheral blood of standard quality can be collected which majorly depends on the collection protocol.

\section{Saliva}

The specimen of saliva is commonly used in clinical diagnostics and forensic toxicology. 
Saliva constitutes various enzymes like salivary amylase and salivary lipase. Appropriate storage and preservation of saliva is very important for the stability of saliva constituents. It has been seen that the stability of salivary amylase and other enzymes do not show a significant fall when store for longer duration of time. Normally saliva is used to estimate drug concentration as well as in diagnostic purposes.

\section{Preservation and storage}

Various parameters affect the specimen quality and quantity at the time of storage like temperature and pressure. Storage of specimen generally depends on the duration of storage. For short term storage specimen are kept in -4 degree $\mathrm{C}$ while for long term storage ( 2 weeks) sample are stored in 20 degree $\mathrm{C}$.

Blood sample are mostly preserved with additives like sodium fluoride $2 \% \mathrm{w} / \mathrm{v}$. In order to prevent the coagulation of blood potassium oxalates are also used as additives during preservation. In order to resist the microbial infection, sodium fluoride is commonly used as additive.

It has been experimentally proved that for shorter period of time saliva can be stored at 4 degree Celsius while for longer duration of time (6 months) it can be stored at -20 degree Celsius.

\section{Effect of storage conditions on the quality of the RBC units}

Storage injuries are generally names that include all the changes that occur as red blood cells age in storage solutions. These changes cause hemolysis (concurrent increase in extracellular free iron, heme, and hemoglobin and decreased biological activity due to nitric oxide [NO] entrapment), morphological changes, lactate and potassium/calcium accumulation, 2 There will be a decrease. 3-DPG and ATP, reduced $\mathrm{pH}$ and glycolysis, and accumulation of released bioactive proteins, lipids, and microparticles or microvesicles derived from red blood cells (MP/MV). This classified the RBC units as "young" $(<14-$ 21 days) and "old" (>21 days). Recent studies have shown a significant impact of storage time and manufacturing procedures on the growth of cell-free mitochondrial DNA (mtDNA) and extracellular vesicles (EV) in the red blood cell unit. Although the clinical relevance of these findings is unknown, both mtDNA and EV may affect the safety of the transfusion. This is because they represent a damageassociated molecular pattern (DAMP) that can stimulate the recipient's immune system.

Accumulation of bioactive lipids during red blood cell storage has been identified as a possible cause of posttransfusion sequelae in vulnerable populations. Metabolomics studies aimed at quantifying a panel of bioactive lipids in the leuko reduced (LR) and non leuko reduced red blood cell (NLR) units during storage showed that leuko reduction significantly increased bioactive lipid production. It has been shown to be dimmed. However, despite leukapheresis, the main polyunsaturated fatty acids (PUFAs) and their oxidation products (oxylipines) were observed in the red blood cell units stored for 42 days.

Numerous clinical research ventures assess resistant segments as biomarkers that mirror the patient's wellbeing and the result of the mediation. The intrinsic and versatile insusceptible segments are available in the blood and saliva and are alluring focuses for biomarkers. Blood is commonly viewed as the liquid of decision for assessing fundamental procedures. Be that as it may, blood inspecting conveys potential dangers for the subject, for example, transient distress, wounding, venipuncture site diseases, and frailty (when enormous sums are required or when the subject is helpless). Blood inspecting is likewise less preferred in examines including youngsters and other research subjects with troublesome venous access (i.e., the old or basically sick). Latent slobbering tests are viewed as the highest quality level for unstimulated saliva examination, however gathering and putting away saliva on channel paper is progressively advantageous. No examination of cytokine levels in saliva tests acquired by these two strategies has been distributed. This methodological examination contrasted numerous blood biomarkers and saliva tests gathered from volunteers in two distinct manners.

\section{Review of literature}

Karinen (2010) ${ }^{[1]}$, venipuncture are used to collect ante mortem blood. The procedure of collecting blood includes usage of syringe to take blood from arm and before that collection site is swipe with antiseptic solution. Collection of post mortem blood is quite different because of the viscous nature of blood and presence of blood clots. Normally in ante mortem specimen $10-20 \mathrm{ml}$ of blood and $1-5 \mathrm{ml}$ of saliva is taken for forensic analysis while in post mortem specimen, $25 \mathrm{ml}$ of blood is taken for forensic toxicological investigation.

Jones (2007) ${ }^{[11]}$, from pericardial sac, central blood is collected. This procedure includes the insertion of needle through chest wall. This may result in contamination of blood as blood from arteries and veins get mixed.

Anderson (1990) ${ }^{[15]}$, collection of cardiac blood sample in post mortem cases may show elevated concentration of drug in blood sample.

Crouch (2005) ${ }^{[4]}$, in various trauma and brain tumor cases, clots presents inside the cranial nerve show drug concentration hence help in forensic toxicological investigation. Various peripheral blood specimen can be used in post mortem testing but among all specimen femoral is the most widely used source of blood specimen in post mortem cases as it can be used everywhere in forensic analysis. Various factors influence the concentration of drug in plasma saliva which basically complicates the pharmacological interpretation.

Kidwell (1998) ${ }^{[3]}$, there are various methods used to collect saliva or oral fluid used in clinical labs and forensic analysis. Among them saturation of absorbent swab is most commonly used to take saliva sample. Salivary gland of our body produces 1.5 litres of saliva every day. For drug examination mixed saliva is used in various lab testing. Sample handling is important in forensic toxicology as there may be time gap between collection of specimen and analysis of specimen. Preservation of sample in appropriate medium is very important in forensic toxicology as inappropriate preservation show negative qualitative and quantitative results during forensic analysis.

Kavert (2001), different additives are used in commercially available test tubes depending on which laboratory it is going to be use. Commonly used additives in commercially available blood tubes are EDTA, heparin etc. Various antioxidants like ascorbic acid $0.24 \% \mathrm{w} / \mathrm{v}$ are commonly used to lower down oxidative losses.

Skopp \& Potsch (2004) ${ }^{[2]}$, sodium azide is also used as 
preservative agent but if the sample is for immunological assay it should not be used. Mostly sodium fluoride is used as additive in blood sample preservation in both ante mortem and post mortem cases but in some specific cases it accelerate chemical degradation. If blood sample or other specimen contain lysogenic acid then it should be stored in foil covered with container and should be placed at a place away from sunlight.

J hardwick (2008) [9], suggest specific temperature to store whole blood sample varies from 4 degree $\mathrm{C}$ to -2 degree Celsius. Red blood cells are placed in refrigeration immediately after collection at 4 to -20 degree C. Platelets should be stored at 22 to -2 degree Celsius. Plasma should be stored at colder temperature i.e. -25 degree Celsius. He further added that various equipment's are used to store blood samples. Among them blood storage refrigerator are most commonly used as it maintain whole blood and its constituent stability at 4 to -2 degree Celsius.

Dos Santos (2018) \& Karim (2013) ${ }^{[5,8]}$, stated that the specimen of saliva is commonly used in clinical diagnostics and forensic toxicology. Saliva constitutes various enzymes like salivary amylase and salivary lipase. Appropriate storage and preservation of saliva is very important for the stability of saliva constituents. It has been experimentally proved that for shorter period of time saliva can be stored at 4 degree Celsius while for longer duration of time (6 months) it can be stored at -20 degree Celsius. Analytes of saliva don't show significant changes with storage temperature. Moreover there are certain enzymes of saliva like lipase and $\mathrm{Ck}$ which are highly stable at -80 degree Celsius.

Dr. Amit gaur ${ }^{[12]}$ in his study stated that while storing blood and saliva for clinical and forensic testing, there are various physical and biochemical effects of storage on blood and its components. It includes the effect of temperature on red blood cell metabolism and survival, use of anticoagulant and its effect on viability of red blood cells, $\mathrm{pH}$ of cell and microbial infection prevention.

Kanchagar C (2014) ${ }^{[16]}$, researched the impact of capacity time and capacity temperature of entire blood on the nature of DNA and RNA. For RNA, a capacity time of as long as 24 hours is significant for gathering excellent RNA samples for NGS tests. Capacity times of as long as 32 hours are worthy when microarray examines are arranged. Additionally, capacity at low temperatures doesn't forestall debasement of RNA samples. In particular, putting away entire blood samples in a cooler drastically harms RNA samples. When put away in entire blood, DNA is safe inside 15 days, yet WBC lysis drastically lessens the measure of DNA. What's more, over a time of three days, it fundamentally alters the DNA methylation status internationally and locally. Our discoveries give a benchmark to gathering top notch DNA and RNA from blood samples.

Veskoukis AS (2015) ${ }^{[6]}$, stated that salivary organs have a rich vascular framework wherein saliva is sifted and prepared. The segments of saliva can be gotten completely from the salivary organs, or can be gotten from the blood by uninvolved dissemination or dynamic vehicle. In the event that the segments of saliva are gotten from the blood, the degrees of the biochemical and immunological segments estimated in the saliva may mirror the levels in the blood. Replacement of a saliva blood test for biomarker examination is significant in light of the fact that saliva assortment is less obtrusive and there is no hazard related with blood assortment.

\section{Conclusion}

When the biological sample like blood is stored for a longer duration of time then various additives are used in order to maintain the fragility of blood cells. In forensic science glass tubes are generally used for ante mortem and post mortem blood sampling. Different additives are used to stabilize the blood specimen in these glass tubes like sodium fluoride. Easiness of using and handling the specimen, its volume and putrefactive etc. are some of the parameter that should be considered while selecting specimen.

Storage and preservation of blood mainly depends upon the duration of storage. When blood sample is stored in cold temperature then anti-coagulant agents, anti-microbial chemicals etc. are added in the sample test tube. In higher temperature crystal are formed in the cell result in death of cells so various chemical like glycerol in appropriate concentration are added in the blood test tube. Saliva is produced by salivary gland in our oral cavity. Saliva contain various enzyme that act as biomarker to estimate various chemical concentration in a suspect body in a criminal investigation. For short term storage specimen are kept in -4 degree $\mathrm{C}$ while for long term storage ( 2 weeks) sample are stored in -20 degree $\mathrm{C}$.

\section{References}

1. Karinen $\mathrm{R}$ et al. Comparison of ethanol and other drugs of abuse concentrations in whole blood stored in Venoject glass and plastic and Venosafe plastic evacuated tubes. J Anal Toxicol 2010;34:420-428.

2. Potsch L, Skopp G. Stability of opiates in hair fibers after exposure to cosmetic treatment. Forensic Sci Int 1996;81:95-102.

3. Kidwell DA et al. Testing for drugs of abuse in saliva and sweat. J Chromatogr B Biomed Sci Appl 1998;713:111-135.

4. Crouch DJ. Oral fluid collection: the neglected variable in oral fluid testing. Forensic Sci Int 2005;150:165-173.

5. Dos Santos DR, Souza RO, Dias LB, Ribas TB, de Oliveira LCF, Sumida DH et al. Arch Oral Biol 2018;85:160-5. 10.1016/j.archoralbio.2017.10.016 [PubMed] [CrossRef] [Google Scholar]

6. Margaritelis NV, Veskoukis AS, Paschalis V, Vrabas IS, Dipla K, Zafeiridis A et al. Blood reflects tissueoxidative stress: a systematic review. Biomarkers 2015;20(2):97-108. Epub 2015/01/15. https://doi.org/10.3109/1354750X.2014.1002807 PMID: 25582635.

7. Kuo HC, Li SC, Guo MM, Huang YH, Yu HR, Huang FC et al. Genome-Wide Association Study Identi-fies Novel Susceptibility Genes Associated with Coronary Artery Aneurysm Formation in Kawasaki Dis-ease. PLoS One 2016;11(5):e0154943. Epub 2016/05/14.

8. Kasim NA, Ariffin SHZ, Shahidan MA, Abidin IZZ, Senafi S, Jemain AA et al. Stability of lactate dehydrogenase, aspartate aminotransferase, alkaline phosphatase and tartrate resistant acid phosphatase in human saliva and gingival crevicular fluid in the presence of protease inhibitor. Arch Biol Sci 2013;65:1131-40. 10.2298/ABS1303131K [CrossRef] [Google Scholar]

9. Hardwick J. The Author. Journal compilation. 
Blackwell Publishing Ltd 2008.

10. Clauwaert KM et al. Stability study of the designer drugs 'MDA, MDMA and MDEA' in water, serum, whole blood, and urine under various storage temperatures. Forensic Sci Int 2001;124:36-42.

11. Jones, Coe JI. Postmortem chemistry of blood, cerebrospinal fluid, and vitreous humor. Leg Med Annu 1976, P55-92.

12. Dinis-Oliveira RJ et al. Collection of biological samples in forensic toxicology. Toxicol Mech Methods 2010;20:363-414.

13. Skopp G. Preanalytic aspects in postmortem toxicology. Forensic Sci Int 2004;142:75-100.

14. Escribano D, Contreras-Aguilar MD, Tvarijonaviciute A, Martínez-Miró S, Martínez-Subiela S, Cerón JJ et al. Stability of selected enzymes in saliva of pigs under different storage conditions: a pilot study. J Vet Med Sci 2018;80:1657-61.

15. Prouty RW, Anderson WH (1990). The forensic science implications of site and temporal influences on postmortem blood-drug concentrations.

16. Ward J, Kanchagar C, Veksler-Lublinsky I, Lee RC, McGill MR, Jaeschke $\mathrm{H}$ et al. Circulating microRNAprofiles in human patients with acetaminophen hepatotoxicity or ischemic hepatitis. Proc Natl Acad Sci USA 2014;111(33):12169-74. Epub 2014/08/06. 